\title{
Insect diapause - stability and change
}

from A.D. Lees

GEOGRAPHICAL strains of insects often differ substantially in their diapause characteristics. The critical photoperiod required for diapause induction, the interactions between day length and temperature and the intensity (duration) of dormancy are particularly variable. When the area of distribution extends continuously over a wide range of latitudes these characters form a northsouth cline with critical photoperiod and intensity diminishing towards lower latitudes and diapause sometimes fading out entirely in the sub-tropics. Following the classical work of Danilevsky (Ent. Obozr. 36, 6; 1957), this has been accepted as evidence that geographical strains are closely adapted in their phenology (date of entry into diapause, voltinism etc.) to their own part of the geographical range of the species. Since photoperiod and temperature, as the main agents controlling diapause, vary systematically with latitude, the insect would receive a false indication of season in other parts of the range. This is expected to limit spread along a northsouth axis and might have severe initial effects on introduced species, although some later readjustment by natural selection might well be looked for. In this connection, crosses between different strains have shown that quantitative differences in diapause-related characters

A.D. Lees is Professor of Insect Physiology, Imperial College, London. such as critical photoperiod and diapause intensity depend on polygenic inheritance. Nevertheless, as emphasised by Tauber and Tauber (Evolution of Insect Migration and Diapause p.53-71, Springer-Verlag, 1978), these features can sometimes be modified quite rapidly by directional selection in the laboratory.

Introduced pests that fail to gain a foothold usually escape notice. For this reason the diapause status of the more successful invaders is especially worthy of scrutiny. In this issue of Nature (p.489) Goldson and Emberson show that the Argentine grass stem weevil Hyperodes bonariensis, introduced into New Zealand more than $\mathbf{5 0}$ years ago, has a remarkably stable photoperiodically controlled adult diapause, populations collected in areas extending over $8^{\circ}$ of latitude having much the same critical photoperiod. It seems that the beetle might have achieved multivoltinism (and even greater destructiveness?) if diapause had been eliminated by selection. The absence of diapause in most indigenous New Zealand insects suggests that this condition is not required for winter survival.

In contrast, the photoperiodically controlled winter diapause of the Colorado potato beetle is well suited to its new environment in continental Europe. Introduced into France in 1922, Leptinotarsa has since spread eastwards as far as the USSR, occupying only slightly wider latitudinal limits than in $\mathbf{N}$. America. It seems likely that the dispause control mechanisms and the voltinism have again remained relatively stable (de Wilde, Soc. exp. Biol. Symp. 23, 263; 1969). The pink bollworm Pectinophora gossypiella, originating in India, is a pest which has been transported by commerce to virtually every cotton growing area in the world, mainly during the present century. The geographical strains tested by Ankersmit and Adkisson ( $J$. Insect Physiol. 13, 553; 1967) were found to have very similar critical photoperiods for diapause induction even though the populations came from widely separated latitudes (for example, El Paso $32^{\circ} \mathrm{N}$ and Colombia $3^{\circ} \mathrm{N}$ ). However, many fewer insects from lower latitudes entered diapause at all, even in short days. In this species adaptive changes are already far advanced. In the cornborer Pyrausta nubilalis the evidence suggests that changes in the diapause status are progressing rapidly. Introduced into $\mathrm{N}$. America from Europe before 1920, there has been a strong tendency for the univoltinism of populations in the north to give way to a bi- or multivoltine life cycle in the warmer states of Kansas and Missouri. According to the analysis of Beck and Apple (J. econ. Ent. 54, 550; 1961), these changes involve both photoperiod and temperature control of the inductive daylength as well as more indirect effects of temperature on the rate of larval development. From these few examples it is clear that the adaptive changes shown by different insects are liable to be varied and complex.

advances in raising public awareness of earthquake hazards and in preparing the public for earthquake emergencies. Participants at the meeting were vividly reminded of the sociological and economic importance of earthquake prediction by a series of Japanese films on the disastrous effects of major earthquakes and a Chinese video-tape showing the destruction caused by the 1976 Tangshan earthquake.

Large earthquakes occur in cycles which are long compared to the instrumental records available of seismicity and related geophysical parameters. The seismic cycle appears even longer when viewed in terms of the relatively short time during which data have been gathered specifically for earthquake prediction purposes. Even within the limitations of existing data, however, the meeting clearly showed that consistent patterns are emerging. As data gathering and interpretation improve, the challenges of the next few years will be to identify the techniques which are the most reliable; to test the significance of predictions based on those methods; and to integrate various techniques into an increasingly reliable procedure for earthquake prediction.
In short-term predictions, water-related effects are especially common. A number of papers described the observation of changes 